\title{
EXAMEN NACIONAL DE ENFERMERÍA EN CHILE: IMPORTANCIA Y DESAFÍOS
}

\author{
NATIONAL EXAM OF NURSING IN CHILE: \\ THE IMPORTANCE AND CHALLENGES
}

\author{
Angela Castellano Salas * \\ Cristina Tagle Vargas ** \\ Luz Galdames Cabrera ${ }^{* * *}$ \\ Náyade Riquelme Pereira ${ }^{* * * *}$ \\ CeCilia Landman NaVARro ${ }^{* * * * *}$ \\ Sonia Peroni Parraguez ${ }^{* * * * *}$
}

\begin{abstract}
RESUMEN
Si bien la evaluación de la calidad de la formación de los egresados, a través de un examen nacional, es una realidad reciente en Chile, la experiencia internacional es amplia y variada. Este artículo da a conocer la necesidad y trascendencia de aplicar algún tipo de medición de competencias en egresados de carreras de enfermería, las características de las evaluaciones que realizan algunos países que las han implementado; así como las políticas y organizaciones que regulan su aplicación. También describe la experiencia de aplicación de un examen nacional en el área de la salud en Chile, como es el caso de medicina y enfermería.
\end{abstract}

Palabras clave: Educación en enfermería, evaluación educacional, estudiantes de enfermería.

\begin{abstract}
Assessing the quality of education of graduates, through a national exam, is a recent activity in Chile, however, international experience is extensive and varied. This article shows the need and importance of some form of measurement of skills in graduates of nursing careers, the characteristics of the assessments made by some countries that have implemented it, as well as policies and organizations regulating its implementation. It also describes the experience of implementation of a national exam in the area of health in Chile, such as medicine and nursing.
\end{abstract}

Key words: Education, nursing, educational measurement, students.

Fecha recepción: 21/01/2011 Fecha aceptación: 18/04/2011

\footnotetext{
*Enfermera. Docente Pontificia Universidad Católica de Chile, Chile. Email: acastell@uc.cl

${ }^{* *}$ Enfermera. Docente Universidad de Los Andes, Santiago, Chile. Email: mctagle@miuandes.cl

${ }^{* * *}$ Enfermera. Docente Universidad Andrés Bello, Santiago, Chile. Email: lgaldames@unab.cl

${ }^{* * * *}$ Enfermera. Docente Universidad de Concepción, Concepción, Chile. Email: nriquelm@udec.cl

${ }^{* * * * *}$ Enfermera. Docente Universidad de Valparaíso, Valparaíso, Chile. Email: cecilia.landman@uv.cl

****** Enfermera. Licenciada en Educación, Chile, Email: soniaperoni@yahoo.com

Comisión Examen Nacional de Enfermería.
} 


\section{INTRODUCCIÓN}

Desde su creación en el año 1962, la Asociación Chilena de Educación en Enfermería (ACHIEEN) ha tenido la misión esencial de velar por la calidad de la formación de los egresados de carreras de enfermería en Chile. Es así como a finales de los 80 se trabajó arduamente en el establecimiento de criterios de calidad para la incorporación de carreras a la Asociación, y en la década siguiente, la comisión curricular de dicho organismo elaboró el documento "Modelo de Acreditación de Escuelas de Enfermería y EnfermeríaObstetricia”. Esta comisión estuvo conformada por académicas de la Universidad de Valparaíso, Universidad de Chile y Pontificia Universidad Católica, y el documento fue considerado posteriormente como un referente para la elaboración de los criterios específicos de acreditación de carreras de enfermería, por parte del comité técnico de la Comisión Nacional de Acreditación de Pregrado (CNAP), hoy Comisión Nacional de Acreditación (CNA) (1).

Sin embargo, esta función se ha complejizado en forma importante desde la década del 90 en adelante, debido al surgimiento creciente de nuevos programas y escuelas de enfermería en el país, derivado de las normativas de 1980 (2) sobre la educación superior y de la Ley Orgánica de Calidad de la Educación (LOCE) (3), que otorgan autonomía a las instituciones de educación superior para ofrecer programas de pregrado y postgrado. En ese contexto educacional, se establece en el año 2006 el Sistema Nacional de Aseguramiento de la Calidad (4), que regula, entre otros temas, el licenciamiento y la acreditación de la calidad de las instituciones, carreras o programas de educación superior. Esta autonomía académica, económica y administrativa de la educación superior es regulada por el Consejo Superior de Educación y ha sido mantenida por la Ley General de la Enseñanza en Chile (LGE) (5), del año 2009.
Sobre el tema, autores colombianos refieren que la autonomía debería entenderse como la libertad para prestar un servicio educativo de alto nivel académico y administrativo, pero acompañada de la responsabilidad para asumir las consecuencias de las acciones ejecutadas y de la voluntad del permanente rendimiento de cuentas a la sociedad y al Estado (6).

La creación de nuevos programas y carreras de enfermería se relaciona también con el déficit de enfermeras en Chile y a nivel global. Según datos otorgados por el Colegio de Enfermeras AG, el déficit de este recurso profesional en el año 2000 equivalía a 4.000 enfermeras, cuando el requerimiento en el sector público de atención de salud debería ser de 8.000 enfermeras para proporcionar una atención mínima indispensable, es decir, aquella que no puede ser proporcionada más que por este profesional. Así, esta asociación gremial plantea que el déficit de enfermeras en la última década ha tenido consecuencias para la atención en salud, las que afectan en mayor medida al sector público (7).

Esta situación ha provocado un gran interés de las instituciones formadoras por impartir esta carrera. Si en el año 2000 había 18 universidades en el país que ofrecían 18 programas de formación de enfermeras; al año 2010 ya existían 38 instituciones de educación superior que impartían 110 programas, lo que representa el incremento de un $61 \%$ en la última década $(8,9)$. Por otra parte, la carrera de enfermería se ubica como la segunda profesión más elegida por los postulantes que acceden a la educación superior, en vista de las oportunidades y ofertas laborales aseguradas (8).

Al respecto, existe preocupación por parte de la ACHIEEN sobre la heterogeneidad observada en los diversos proyectos educativos que existen actualmente, unido al hecho que sólo el 17,2\% del total están actualmente acreditados. La importancia de este hecho radica en la necesidad del país de contar con profesionales de enfermería que cumplan las 
competencias para proporcionar cuidados de calidad, seguros y costo-efectivos. Los criterios de calidad para las instituciones que forman enfermeras, así como las competencias generales y específicas que deben cumplir sus egresados (perfil de egreso), están definidas a nivel nacional por la Comisión Nacional de Acreditación (CNA) y son las que rigen en los procesos de acreditación por parte de dicho organismo u otras agencias $(1,10)$.

Los antecedentes expuestos permiten identificar varios factores que deben conjugarse en pos del bien común. Por una parte, se debe responder a las demandas sociales con más y mejores egresados, como también lograr una incorporación exitosa de los nuevos profesionales al mercado laboral; y lo más relevante, es que ellos sean capaces de asumir en forma competente la gestión del cuidado de las personas, grupos y comunidades, como un acto exclusivo de la enfermera. Por ello, es relevante evidenciar las competencias de los egresados de la carrera de enfermería mediante adecuadas estrategias de evaluación, en la cual convergen múltiples aspectos de la organización universitaria en su conjunto, con importantes repercusiones en la calidad del proceso educativo (11).

En este contexto, la ACHIEEN se propuso en el año 2004 la misión de cautelar la calidad de la formación en las carreras de enfermería, a través de la medición de los conocimientos básicos e indispensables que deben estar presentes en los egresados de la carrera (área del saber), de su aplicación en el cuidado de enfermería (área del saber hacer), así como del enfrentamiento a las situaciones de salud de las personas, en un marco de principios y valores (área del ser) y en un contexto ciudadano y social (aprender a convivir) (12). Si bien las preguntas de este examen fueron diseñadas acorde a los requerimientos del perfil profesional, por ahora, están fundamentalmente orientadas hacia la medición del área cognitiva y su aplicación a la práctica. A esta iniciativa de la Asociación se sumó el interés del Colegio de Enfermeras AG de Chile por esta materia, con el fin de garantizar a la población cuidados de enfermería de calidad.

En este artículo se presenta el marco referencial e importancia de la aplicación del examen nacional de enfermería en egresados de carreras de Enfermería en Chile. Se mencionan dos modalidades de medición de competencias utilizadas en países extranjeros con trayectoria en el tema (EE.UU. y Canadá), así como la experiencia del examen nacional de medicina y enfermería en nuestro país. Por último, los comentarios y desafíos a futuro sobre el tema.

\section{MARCO REFERENCIAL}

Las actuales tendencias de la educación universitaria exigen a las instituciones de educación superior el diseño de estrategias y procesos orientados a incrementar la calidad de la formación, a mejorar el aprendizaje de los estudiantes, y a reducir la reprobación y el abandono, para lograr índices de aprovechamiento y de eficiencia terminal satisfactorios (11). En Chile estos aspectos corresponden a indicadores de eficiencia académica.

El psicólogo David McClelland, de la Universidad de Harvard, argumentó que los exámenes académicos tradicionales no garantizan el desempeño en el trabajo ni el éxito en la vida $y$, frecuentemente, discriminan a las minorías étnicas, las mujeres y otros grupos vulnerables en el mercado laboral. Plantea, entonces, la necesidad de encontrar otras variables, tales como las competencias, las que podrían predecir cierto grado de éxito o, al menos, cometer menos errores (13).

$\mathrm{Al}$ respecto se puede mencionar el trabajo de Miller $(14,15)$, quien desarrolló un modelo de competencia profesional representado por una pirámide compuesta de varios niveles de complejidad. En la base se sitúan los conocimientos (el saber), sobre los que 
se apoya la competencia (el saber cómo). A un nivel superior se encuentra el desempeño (mostrar cómo) y finalmente la acción en la práctica real (el hacer). Este modelo resume bien el constructo de la competencia y permite operacionalizar su evaluación y en particular, la elección de los instrumentos de medida. La complejidad del proceso explicita que el hecho de tener conocimientos no necesariamente significa saber cómo utilizarlos o saber cómo desempeñarse con sabiduría y profesionalismo.

Una de las definiciones más utilizadas para comprender el concepto de competencia se refiere al "conjunto de aptitudes que representan una combinación dinámica de atributos (con respecto al conocimiento y su aplicación, a las actitudes y a las responsabilidades) que describen los resultados del aprendizaje de un determinado programa o cómo los estudiantes serán capaces de desenvolverse al finalizar un proceso educativo" (16). Se refiere a un conjunto identificable y evaluable de conocimientos, actitudes, valores y habilidades relacionados entre sí, que permiten desempeños satisfactorios en situaciones reales de trabajo, según estándares utilizados en el área ocupacional (17).

La definición de Kane es dinámica e interesante. Este autor define la competencia profesional, para un individuo dado, como: “...el grado de utilización de los conocimientos, las habilidades y el buen juicio asociados a la profesión, en todas las situaciones que se pueden confrontar en el ejercicio de la práctica profesional" (18).

En esa línea, la clasificación de competencias que ha establecido el Proyecto Tuning Europa y Latinoamérica (11) y también la CNA (1), ha sido en dos órdenes: genéricas o generales y específicas o profesionales. Las primeras se refieren a los componentes instrumentales y sistémicos relevantes para preparar a los estudiantes para su futuro rol en la sociedad, en términos de empleabilidad y de capacidades ciudadanas, sin importar la carrera que seleccionen; en cambio las com- petencias específicas se definen como propias de cada profesión, y ambas orientan el proceso formativo (12).

\section{Experiencias de medición de competencias en enfermería}

La experiencia internacional indica que desde hace varios años se utilizan distintos métodos para evaluar competencias, tanto para certificar la formación conducente a algún título profesional como para la reacreditación profesional. Si bien es cierto existen variadas experiencias en países de habla inglesa y Latinoamérica, se citarán las de EE.UU. y Canadá, dada su trayectoria y porque sus tipos de evaluación responden en mayor medida a los intereses de lo que se está trabajando en el examen de enfermería en Chile.

En los Estados Unidos, el Consejo Nacional de Juntas Estatales de Enfermería determinó que los requisitos mínimos para obtener la licencia profesional de enfermería, necesaria para ejercer como enfermera registrada (RN) y la licencia de enfermera práctica (LPN), son haber cursado un programa acreditado por el Estado y aprobar el examen National Council Licensure Examination (NCLEX). Este examen mide el nivel de competencias, a través de preguntas de opción múltiple, en un sistema interactivo denominado Pruebas de Adaptación Computarizada (CAT), que enfrentan al examinado a diferentes escenarios simulados. Las preguntas se centran en la capacidad para tomar decisiones en diversos contextos de la atención al paciente en condiciones críticas, considerando la calidad y seguridad de la gestión del cuidado. Los contenidos del examen se relacionan con las necesidades del cliente, categorizados en áreas y sus respectivos subtemas, tales como: gestión de la atención; seguridad y control de infecciones; promoción y mantención de la salud; adaptación fisiológica; medio ambiente y cuidado eficaz, entre otros (19).

En Canadá existe el Canadian Registered Nurse Examination (CRNE), examen obli- 
gatorio para obtener el título de enfermera ante el Estado de Canadá que tiene el propósito de proteger al usuario, asegurando que la enfermera posee las competencias necesarias para la práctica segura y efectiva. También se espera que las enfermeras utilicen el conocimiento y la investigación para construir una cultura basada en la evidencia. El nivel de competencia de las enfermeras registradas en todas las provincias y territorios es evaluado por el CRNE, excepto en Quebec, donde deben realizar un examen para obtener la licenciatura, y un porcentaje de la evaluación es realizada por el CRNE. El dominio de contenido para este examen está definido en un marco de 148 competencias, organizadas en cuatro categorías: principios éticos; colaboración enfermera-cliente; salud y bienestar, y cambios en la salud. Este examen pretende medir en los egresados la aplicación del conocimiento teórico en diversos contenidos y principios de enfermería y además, deben demostrar habilidades en la aplicación del conocimiento en un contexto de escenarios simulados. La Asociación de Enfermeras de Canadá desarrolla y administra este examen en colaboración con las autoridades reguladoras territoriales o provinciales. Dicha entidad define la práctica profesional de la enfermera, como aquella que requiere un juicio profesional, conciencia política y responsabilidad social, capacidad de gestión, trabajo en equipo, liderazgo y desarrollo profesional continuo (20).

La primera experiencia en Chile de medición de competencias en egresados de carreras de la salud fue en la carrera de medicina, a través del Examen Médico Nacional (EMN). Las facultades de medicina que constituían la Asociación de Facultades de Medicina de Chile (ASOFAMECH) implementaron, a comienzos del 2000, un examen final común para ser rendido por todos los egresados de las carreras de medicina. Durante los años 2001 y 2002 se realizaron dos versiones piloto y la primera aplicación oficial se realizó el 17 de diciembre de 2003, aplicándose anual- mente desde entonces. El EMN surgió por la necesidad de contar con una observación objetiva de los conocimientos teóricos de los egresados, al momento de su titulación profesional. En parte, fue la respuesta frente a la aparición de variadas escuelas de medicina, con distintas orientaciones respecto a sus énfasis y perfiles de egreso, con el objetivo de una entrega uniforme y mensurable de los conocimientos más propios e irrenunciables, y por otro lado, se planteó como una medida tendiente a asegurar la calidad de la educación médica. Así, este examen constituye además un elemento que permite dar confianza ante la opinión pública, puesto que mide si los egresados cumplen con los objetivos definidos y acordados del perfil profesional (21). El EMN fue un examen voluntario creado por las facultades de medicina para seleccionar postulantes a sus programas de postítulo y durante su administración, ASOFAMECH subvencionó el costo del examen.

Posteriormente surgió el Examen Único Nacional de Conocimientos de Medicina (EUNACOM) (22), como una medición de conocimientos y habilidades clínicas, exigido para acceder a cargos médicos y programas de especialización financiados por el estado, así como para firmar convenios con el Fondo Nacional de Salud (FONASA). Dicho examen se transforma entonces en una obligación legal, cuyo beneficio es personal (cargos, becas, convenios FONASA y habilitación profesional) y los responsables del pago son los individuos. El diseño y administración de este examen está regulado por la Ley 20.261 y su diseño y administración fue encargado por el Ministerio de Salud a ASOFAMECH en agosto de 2009. El consejo de decanos de esa asociación delega la administración del examen en el director del EUNACOM, quien cuenta con la asesoría de un consejo de directores y de un consejo estadístico.

La segunda experiencia chilena en el área de la salud fue la de enfermería. Como ya se planteó anteriormente, la ACHIEEN encomendó, en el año 2004, a la Comisión Exa- 
men Nacional la tarea de establecer un sistema nacional de evaluación de los egresados de las carreras de Enfermería, denominado Examen Nacional de Enfermería (ENENF) (23), lo que significaba asumir la responsabilidad en el diseño del instrumento, en su aplicación y en la evaluación de sus resultados. Esta Comisión fue conformada por cinco integrantes nominados por el directorio de la asociación, los que debían acreditar una trayectoria académica en función de la tarea encomendada. También se constituyó un Comité Técnico, integrado por un representante de cada unidad académica asociada, con la función de colaborar en las distintas etapas del proceso, especialmente en su calidad de expertos en las áreas de contenidos disciplinares del examen ${ }^{1}$.

Durante los años 2004 al 2007 se trabajó principalmente en la revisión bibliográfica sobre modelos teóricos, metodologías e instrumentos de evaluación de competencias de egreso, considerando la realidad nacional e internacional. La experiencia del examen médico nacional también fue un referente importante considerado en este estudio, así como las entrevistas con expertos en el tema. Lo anterior favoreció la continua capacitación de los miembros de la comisión y su socialización con el comité técnico a través de talleres. Otra tarea fue la construcción de las matrices de contenidos, consensuadas por las 18 escuelas asociadas y la elaboración de preguntas que constituirían el banco de ítemes del examen. En el año 2006 se contó con la asesoría de un experto en educación y en el mes de diciembre del 2007 se aplicó la primera prueba piloto, con el fin de validar preguntas $^{2}$.

Después de la evaluación de ese proceso, y en vista de su trascendencia y complejidad técnica, la Comisión determinó que para cumplir con las etapas siguientes de esta medición era necesario contar con la asesoría de un organismo experto en la materia, con el fin de asegurar la calidad del instrumento, así como la seguridad y confiabilidad de su aplicación y procesamiento de los resultados. El estudio de las propuestas (Huarte de San Juan, MIDE UC y U. Valparaíso) para la asesoría técnica del examen y su costo, por parte del directorio de ACHIEEN, lentificó el cronograma fijado por la Comisión. De igual forma se continuó con la revisión de preguntas y la sistematización del trabajo realizado en el período 2008-2009³.

De acuerdo a las disposiciones emanadas del directorio de ACHIEEN y del consejo asesor, en el año 2009 se estableció un convenio de asesoría técnica con el Departamento de Evaluación, Medición y Registro Educacional (DEMRE), dependiente de la Universidad de Chile, teniendo en cuenta su vasta trayectoria en los procesos de evaluación educacional del país. El apoyo de esa entidad se ha referido especialmente a la construcción y validación de preguntas, al diseño, ensamblaje y orientaciones para la aplicación del instrumento, así como al análisis estadístico de los resultados. En vista de la naturaleza del examen, sus implicancias y la complejidad de llevar a cabo una aplicación simultánea a nivel nacional, se elaboró el reglamento del examen ${ }^{3}$, el cual define la estructura, funciones y responsabilidades de cada uno de los participantes del proceso, de modo de asegurar su confiabilidad. En esta tarea se contó con la correspondiente asesoría legal.

Dicho reglamento define el ENENF como una estructura de evaluación de los egresados de carreras de Enfermería, siendo pro-

\footnotetext{
${ }^{1}$ Galdames L, Peroni S, Landman C, Castellano A, Tagle MC. Informe Final Examen Nacional de Enfermería (2004-2008). Comisión Examen Nacional. ACHIEEN.

${ }^{2}$ Galdames L, Peroni S, Landman C, Castellano A, Tagle MC. Informe Final Examen Nacional de Enfermería. Comisión Examen Nacional. ACHIEEN 2008-2009.

${ }^{3}$ Galdames L, Peroni S, Landman C, Castellano A, Tagle MC, Riquelme N. Reglamento Examen Nacional de Enfermería 2009. Comisión Examen Nacional. ACHIEEN.
} 
piedad intelectual de la ACHIEEN. Su objetivo es aportar a la sociedad información que verifique el cumplimiento de objetivos cognitivos comunes a la formación de una enfermera(o) generalista y que deben estar presentes en todas las unidades académicas pertenecientes a la Asociación, conforme a los perfiles que ellas mismas han determinado y consecuentes con los criterios de acreditación establecidos. Asimismo, pretende entregar a las unidades académicas el resultado de su facultad o escuela, de modo de contribuir al mejoramiento continuo de los programas de estudio. El propósito más trascendente de esta medición es asegurar que los egresados posean las competencias disciplinares y profesionales necesarias para otorgar cuidados de calidad a las personas.

Se estipuló inicialmente que a este examen, de modalidad escrita, podrían acceder las unidades académicas (facultades o escuelas de Enfermería) socias de la ACHIEEN, a través de la participación voluntaria de sus estudiantes. Estas escuelas y sus respectivas subsedes corresponden a: U. Arturo Prat, U. Tarapacá, U. de Antofagasta, U. de La Serena, U. Valparaíso, Pontificia Universidad Católica de Chile, U. de Chile, U. de Santiago, U. de Los Andes, U. Andrés Bello, U. Mayor, U. Católica del Maule, U. del Bío Bío, U. de Concepción, U. Santísima Concepción, U. La Frontera, U. Austral, U. de Magallanes (23).

Las áreas temáticas evaluadas en el examen fueron consensuadas por estas unidades académicas, las que han sido procesadas en matrices de contenido ${ }^{4}$, definidas en: cuidados de enfermería en el adulto (incluye adulto mayor, mujer y urgencias); enfermería en salud mental y psiquiatría; cuidados de enfermería del niño y adolescente (incluye urgencias infantiles); enfermería en salud familiar y comunitaria; gestión y liderazgo de enfermería; bases teóricas de la disciplina de enfermería; educación para el cuidado de la salud; investigación en enfermería, y ética en enfermería. La proporción de cada área evaluada se determinó considerando los planes de estudio de las unidades académicas involucradas en el proceso, así como el contexto de salud chileno, los cambios demográficos y las competencias disciplinares y profesionales definidas por la CNA (1).

El trabajo en conjunto con el DEMRE ha permitido realizar ya dos mediciones, en los años 2009 y 2010, las que han tenido como objetivos, probar la capacidad que tiene la asociación para aplicar una prueba a nivel nacional en todas las unidades académicas Asociadas, resguardando estándares de calidad y seguridad y, por otra parte, validar un porcentaje de preguntas, de acuerdo a las áreas disciplinares definidas con estas unidades académicas.

Actualmente la Comisión está sistematizando los resultados de esta experiencia y continúa en el proceso de elaboración y revisión de nuevas preguntas, con el apoyo técnico del DEMRE y la colaboración del comité técnico; en la realización de talleres de construcción de preguntas dirigidos a académicos de las escuelas asociadas, y en la actualización de las matrices de contenidos. El inicio de la versión oficial del examen se implementará cuando se cuente con un banco suficiente de preguntas validadas, así como un sistema operativo confiable y seguro.

\section{CONSIDERACIONES FINALES}

La responsabilidad asumida por la Comisión en el diseño, aplicación y evaluación del examen nacional de enfermería en 18 escuelas a lo largo del país, ha sido posible gracias al compromiso y apoyo de todos los actores involucrados, tales como el directorio de ACHIEEN y su consejo asesor, el comité téc-

\footnotetext{
${ }^{4}$ Comisión Examen Nacional. ACHIEEN. Matrices de Contenido Examen Nacional de Enfermería 2004-2010.
} 
nico, las unidades académicas, la Federación de Estudiantes de Enfermería (FENEECH), los estudiantes y el Colegio de Enfermeras. Además, la difusión lograda en las unidades académicas a nivel regional y Santiago, como en los diversos medios de comunicación, contribuyeron a la socialización de esta nueva responsabilidad asumida por la ACHIEEN $\mathrm{y}$ unidades asociadas.

El trabajo realizado en conjunto con el DEMRE ha permitido realizar ya dos mediciones, en los años 2009 y 2010, mediante las cuales se ha podido demostrar la capacidad que tiene la Asociación para aplicar una prueba a nivel nacional, acorde a estándares de calidad y seguridad. Por otra parte, se está configurando un banco de preguntas en base a los ítemes validados, los que conformarán el examen definitivo.

Los participantes en la aplicación del examen nacional (2009-2010) han valorado la rigurosidad del proceso, en cuanto a las características del instrumento; la organización y logística de la aplicación; el cumplimiento de los acuerdos establecidos en el reglamento; el manejo de la confidencialidad de la información, así como la aceptación de los estudiantes para participar, mediante un consentimiento informado. Se valora, a su vez, el aprendizaje y lecciones aprendidas para las siguientes aplicaciones.

Se ha potenciado el interés de las escuelas en participar en esta tarea, observándose un mejoramiento continuo en los sistemas de evaluación y construcción de preguntas en las unidades académicas participantes, lo cual se visualiza como una ventaja para las siguientes etapas del proceso.

Se destaca la construcción de las matrices de contenido en forma consensuada con las escuelas, indicando representatividad en la selección de los contenidos esenciales del examen. La clasificación de contenidos en áreas, temas y subtemas ha facilitado el proceso de elaboración y selección de ítemes que respondan a los objetivos de la medición y a la distribución de porcentajes para cada tema, dando las bases para la construcción del instrumento final. La actualización permanente de las matrices de contenidos se considera fundamental para la vigencia del examen.

Existe acuerdo que la evaluación de conocimientos al momento del egreso representa un gran avance, sin dejar de reconocer sus limitaciones para la medición de otras competencias consideradas relevantes para el ejercicio del rol profesional. Aún así, se visualiza bastante campo todavía para seguir avanzando en este instrumento en su modalidad escrita, de modo que las respuestas permitan evaluar también el juicio crítico, la toma de decisiones, la priorización, el criterio profesional, entre otras.

El gran desafío entonces es perfeccionar el examen nacional escrito en forma continua y sistemática; aunque sin duda se requiere avanzar también hacia la medición de competencias de los egresados en las áreas del saber hacer y del saber ser, tal como ya se realiza en otros países. Esto se puede alcanzar a través de pruebas computarizadas en ambientes simulados, con el objetivo de asegurar lo más posible una práctica segura y efectiva.

Aun cuando en esta primera etapa el examen sólo ha sido ofrecido a los estudiantes de las unidades académicas pertenecientes a la ACHIEEN, se tiene presente que esta medición podría transformarse, en un futuro no lejano, en un examen abierto para todas las carreras de Enfermería del país.

Se cree, con firmeza, que los procesos de evaluación y acreditación contribuyen en forma efectiva al mejoramiento de la formación y, en consecuencia, al desempeño de los egresados en el campo laboral. Sin embargo, la heterogeneidad de la realidad educativa actual, el insuficiente porcentaje de carreras de Enfermería acreditadas, entre otros factores, ameritan progresar en los sistemas de medición de competencias de egreso, como una responsabilidad social. Por tanto, todos los esfuerzos que se realicen para evaluar el 
cumplimiento del perfil profesional se orientan finalmente al propósito de asegurar a las personas cuidados de enfermería de calidad.

\section{REFERENCIAS}

1. Comisión Nacional de Acreditación [Internet]. Chile. Perfil Profesional Mínimo de la Carrera de Enfermería. Hallado en: http://www.cnachile.cl/. Acceso 7 de marzo 2011.

2. Decreto con Fuerza de Ley No 1 (DFL 1/80). Fecha Promulgación: 30 diciembre de 1980. Establece requisitos para crear universidades privadas. Biblioteca del Congreso Nacional de Chile [Internet]. Hallado en: http://www.bcn.cl/ Acceso 7 de marzo 2011.

3. Ley Orgánica de Calidad de la Educación No 18962 (LOCE). Fecha de promulgación: 7 de marzo de 1990. Biblioteca del Congreso Nacional de Chile [Internet]. Hallado en: http://www.bcn.cl/. Acceso 7 de marzo 2011.

4. Sistema Nacional de Aseguramiento de la Calidad de la Educación Superior. Ley $\mathrm{N}^{\circ}$ 20129. Fecha de promulgación 23 de octubre del 2006. Biblioteca del Congreso Nacional de Chile [Internet]. Hallado en: http://www.bcn.cl/. Acceso 7 de marzo 2011.

5. Ley General de Enseñanza $N^{\circ} 20370$ (LGE). Fecha de modificación 17 de noviembre del 2006. Biblioteca del Congreso Nacional de Chile [Internet]. Hallado en: http://www.bcn.cl/ Acceso 7 de marzo 2011.

6. Giraldo U, Abad D, Díaz E. Bases para una Política de Calidad de la Educación Superior en Colombia [Internet]. Hallado en: http://www.cna.gov.co/1741/ articles-186502_doc_academico10.pdf. Acceso el 5 de enero de 2010.

7. Colegio de Enfermeras AG. Serie: Falencia de Enfermeras año 2000. Sistema pú- blico de atención en salud. 2000.

8. MINEDUC. Sistema Nacional de Información de la Educación Superior (SIES). División de Educación Superior. Ministerio de Educación. Chile. 2010.

9. Asociación Chilena de Educación en Enfermería [Internet]. Boletín No 2 Sept. 2009. p 3. Hallado en: http://www. achieen.cl/bolet/bol0909.pdf. Acceso el 5 enero de 2010.

10. Latrach C, Soto P, González I, Caballero E, Inalaf C. Aseguramiento de la calidad en la formación de las enfermeras desde la perspectiva de los procesos de acreditación nacional. Cienc. enferm. 2009; 15 (2): 79-94.

11. Romo A, Fresán M. Los factores curriculares y académicos relacionados con el abandono y el rezago, En: Asociación Nacional de Universidades e Instituciones de Educación Superior, ANUIES [Internet]. México. Deserción, rezago y eficiencia terminal en las IES. Propuesta metodológica para su estudio. Serie Investigaciones. Colección Biblioteca de la educación superior. Hallado en: http://www.anuies.mx/ servicios/d_estrategicos/libros/lib64/7. html. Acceso el 7 de octubre de 2010.

12. Delors J. La educación encierra un tesoro. Santillana Ediciones UNESCO [Internet]. 1995. Hallado en: http://www. unesco.org/education/pdf/DELORS_S. PDF. Acceso el 7 de octubre de 2010.

13. Vossio R. Certificación y normalización de competencias. Orígenes, conceptos y prácticas. Boletín CINTERFOR [Internet]. No 152. P 51-72. 2002. Competencia laboral y valoración del aprendizaje. Hallado en: http://www.cinterfor.org.uy/ public/spanish/region/ampro/cinterfor/ publ/boletin/152/pdf/vossio.pdf. Acceso el 7 de octubre de 2010.

14. Miller GE. The assessment of clinical skills/competente/performance. Acad Med. 1990; 65 (9 Suppl): S63-7.

15. Durante E. Algunos Métodos de Evalua- 
ción de las Competencias: Escalando la Pirámide de Miller. Revista Hospital Italiano. Buenos Aires. 2006; 26(2): 57-61.

16. Definición dentro del marco del Proyecto Tuning Europa [Internet]. 2005. Hallado en: http://tuning. unideusto.org/tuningeu/. Acceso el 5 de octubre de 2010.

17. Tuning Educational Structures in Europe [Internet]. 2011. Hallado en: http://tuning.unideusto.org/tuningeu/. Acceso el 7 de enero de 2011.

18. Kane M.T. The assessment of clinical competence. Evaluation and the Health Professions. 1992; 15:163-182.

19. All Nursing Schools [Internet]. NCLEX Exam Learn about the National Council Licensure Examination Get State Board of Nursing Info. Hallado en: http://www. allnursingschools.com/faqs/nclex. Acce- so el 7 de enero de 2011.

20. Canadian Nurses Association [Internet]. Apply to be a CRNE Exam Development Participant. Hallado en http://www. cna-nurses.ca/CNA/nursing/rnexamn/ volunteer/default_e.aspx. Acceso el 7 de enero de 2011.

21. Espinoza R. Examen médico nacional y educación médica en Chile. Rev Chil Pediatr 2008; 79(1): 9-12.

22. Examen Único Nacional de Conocimientos de Medicina [Internet]. Hallado en http://www.eunacom.cl. Acceso el 7 enero de 2011.

23. Asociación Chilena de Educación en Enfermería [Internet]. Examen nacional de enfermería. Boletín No1. Junio 2010. p8. Hallado en: http://www.achieen.cl/bolet/ bol0610.pdf. Acceso el 10 de enero de 2011. 